\title{
Terapêutica com doses profiláticas de sulfato ferroso como medida de intervenção no combate à carência de ferro em crianças atendidas em unidades básicas de saúde
}

\author{
Treatment with prophylactic doses of ferrous sulphate in the fight against iron \\ deficiency in children attended in basis health units
}

\author{
Marco A. A. Torres*, Kasue Sato**, Yara Juliano**, Suzana S. Queiroz ${ }^{* * *}$
}

\begin{abstract}
TORRES, M. A. A. et al. Terapêutica com doses profiláticas de sulfato ferroso como medida de intervenção no combate à carência de ferro em crianças atendidas em unidades básicas de saúde. Rev. Saúde Pública, 28: 410-5, 1994. Objetivou-se testar a terapêutica com doses profiláticas de sulfato ferroso no combate à anemia carencial ferropriva, em 620 crianças de 4 a 36 meses de idade, atendidas em duas unidades de saúde do Município de São Paulo, Brasil. As crianças foram submetidas a coleta de sangue para dosagem de hemoglobina. Em seguida, foi prescrito dosagem de $12 \mathrm{mg} / \mathrm{dia}$ de ferro elementar, por 30 dias. Observou-se que $25 \%$ dos menores de 6 meses apresentaram níveis de hemoglobina inferiores a $11,0 \mathrm{~g} / \mathrm{dl}$. As maiores ocorrências de anemia foram detectadas entre os 9 e 23 meses de idade $(50,0 \%)$. Decorrido o prazo, apenas $37,4 \%$ das crianças com anemia e $52,4 \%$ das não anêmicas retornaram para reavaliaçăo. Das 299 que foram reavaliadas, somente $157(52,5 \%)$ receberam a medicação corretamente. A freqüência de hemoglobinas inferiores a $9,5 \mathrm{~g} / \mathrm{dl}$ caiu de $17,1 \%$ no início, para $8,1 \%$ ao final da intervenção. Por outro lado, o percentual de crianças com hemoglobinas superiores a $12,0 \mathrm{~g} / \mathrm{dl}$ subiu de $13,4 \%$, para $33,4 \%$. As que receberam a suplementação férrica de forma correta registraram queda nos índices de anemia sensivelmente maior que a observada naquelas suplementadas de forma incorreta. Concluiu-se que a terapêutica com doses profiláticas de sulfato ferroso, apesar de se mostrar eficiente na recuperação dos niveis de hemoglobina, apresenta sérios entraves do ponto de vista operacional.
\end{abstract}

Descritores: Anemia hipocrômica, prevenção \& controle. Sulfato ferroso.

\section{Introdução}

A anemia nutricional por carência de ferro é, atualmente, a doença carencial de maior prevalência na população infantil $1^{2,22}$, em nosso meio. Pela sua magnitude, redução da atividade física, capacidade de aprendizado e resistência às infecções, bem como sua associação com o risco gravídico, a anemia nutricional aparece como a segunda prioridade, no mundo, entre as doenças carenciais de saúde pública ${ }^{5,15}$.

Na cidade de São Paulo, em pré-escolares, a

* Grupo de Atenção à Nutrição do Centro de Apoio ao Desenvolvimento da Atenção Integral à Saúde e do Núcleo de Nutrição do Centro de Referência de Saúde da Mulher, Nutrição, Alimentação e Desenvolvimento Infantil da Secretaria de Estado da Saúde de São Paulo - São Paulo, SP - Brasil

* Departamento de Medicina Preventiva. Disciplina de Bio-Estatística da Escola Paulista de Medicina - São Paulo, SP - Brasil

*** Departamento de Pediatria da Faculdade de Medicina de Botucatu - Universidade Estadual Paulista/UNESP prevalência vem aumentando. Em 1974, a anemia estava presente em $22,7 \%$ das crianças com idades compreendidas entre 6 e 60 meses, numa amostra representativa da população do município $^{19}$. Em 1984, outro estudo registrou $35,6 \%$ de anêmicos ${ }^{12}$, sendo que as maiores prevalências foram encontradas entre os 6 e 11 meses $(53,7 \%)$ e entre 12 e 24 meses $(58,1 \%)$.

As Unidades Básicas do Setor Público de Saúde (UBS) prestam serviços no atendimento à parcela da população mais carente do ponto de vista socioeconômico e, portanto, a mais vunerável aos agravos nutricionais ${ }^{9}$. Em Recife, Romani ${ }^{17}$ encontrou, em uma UBS, $84,8 \%$ de

e Grupo de Atenção do Centro de Apoio ao Desenvolvimento da Atenção Integral à Saúde e do Núcleo de Nutrição do Centro de Referência de Saúde da Mulher, Nutrição, Alimentação e Desenvolvimento Infantil da Secretaria de Estado da Saúde de São Paulo - São Paulo, SP - Brasil

Separatas/Reprints: M. A. A. Torres - Rua Dr. Augusto de Miranda, 1297 - 05026-001 - São Paulo, SP - Brasil

Edição subvencionada pela FAPESP. Processo 94/0500-0 
crianças com hemoglobina inferior a $11,0 \mathrm{~g} / \mathrm{dl}$, na faixa etária de 6 a 11 meses, e 82,0\% na de 12 a 23 meses de idade. Outro estudo, realizado em amostra representativa das UBS de todo Estado de São Paulo, num total de 2.992 crianças de 6 a 23 meses de idade, constatou uma prevalência de anemia de $59,1 \%{ }^{23}$.

A dosagem da hemoglobina, apesar de ser considerada o exame de escolha para o dianóstico da anemia, em estudos populacionais ${ }^{16}$, identifica somente o último estágio da depleção de ferro no organismo, ficando, portanto, grande parte da população com deficiência desse mineral, sem diagnóstico ${ }^{3,13}$. Hebert ${ }^{7}$ (1987), afirma que a ocorrência da depleção de ferro, nos estágios iniciais, é substancialmente maior que a da anemia propriamente dita.

Frente à magnitude do problema, realizouse o presente estudo, cujos objetivos foram determinar a prevalência da anemia em crianças de duas UBS no Município de São Paulo, e testar a suplementação medicamentosa com sulfato ferroso como medida de intervenção no combate à anemia carencial ferropriva.

\section{Material e Método}

O estudo foi realizado no Município de São Paulo: nos Centros de Saúde de Santa Cecília e do Pari. Fizeram parte da amostra 620 crianças, de 4 e 36 meses de idade, atendidas em consulta médica, dentro da demanda espontânea dessas unidades.

$\mathrm{Na}$ pré-consulta, as mães recebiam informações relativas a anemia nutricional por carência de ferro. Após o atendimento médico, as mães eram entrevistadas e solicitadas a autorizar a coleta de sangue em seus filhos para dosagem da hemoglobina, prova de falcização de hemáceas e eletroforese de hemoglobina. Uma vez coletado o sangue, recebiam um vidro de sulfato ferroso, em gotas e a prescrição de administrar uma dosagem de 10 gotas ao dia (equivalente a 12 $\mathrm{mg} /$ Ferro elementar). Agendou-se um retorno para após 30 dias de administração do medicamento. Na oportunidade, a criança era reavaliada do ponto de vista clínico, antropométrico e submetida a nova dosagem de hemoglobina.

$O$ sangue era coletado por punção venosa; os exames eram realizados em duplicata, no laboratório central do Instituto Adolfo Lutz; as hemoglobinas eram dosadas pelo método da cianometa-hemoglobina ${ }^{6}$, em hemoglobinômetros semi-automáticos calibrados com padrões nacionais e internacinais, segundo às normas do Comitê Internacional de Hematologia ${ }^{8}$. O controle de qualidade foi feito pela leitura dos exa- mes em dois aparelhos. A prova de falcização de hemáceas foi realizada pelo método de observação microscópica de lâmina contendo o sangue com metassulfito de sódio a $2 \%{ }^{1}$ e a eletroforese de hemoglobina, em color-gel e agar-citrado ${ }^{4,11}$.

O critério utilizado para caracterização da anemia foi o proposto pela $\mathrm{OMS}^{15}$, ou seja, dosagem de hemoglobina inferior a $11,0 \mathrm{~g} / \mathrm{dl}$ para as faixas etárias estudadas.

Para a análise dos resultados foram aplicados os testes do Qui Quadrado, para tabelas de assossiação e de contingência ${ }^{18}$; teste de concordância de Kappa, complementado pelo teste de discordância de $\mathrm{McNemar}{ }^{10}$; teste $\mathrm{t}$ para dados pareados ${ }^{20}$.

Foram excluídas da amostra 18 crianças que apresentaram prova de falcização de hemáceas positiva e 3 portadoras de hemoglobinopatias detectadas pela eletroforese de hemoglobina.

\section{Resultados}

A Tabela 1 apresenta a amostra estudada, por faixa etária, em cada uma das unidades de saúde (Sta. Cecília e Pari). Os resultados das duas UBS serão apresentados conjuntamente.

A presença de anemia por faixa etária está representada na Tabela 2 . No total das 620 crianças, $38,4 \%$ apresentaram valores da hemoglobina inferiores a $11,0 \mathrm{~g} / \mathrm{dl}$. A anemia estava presente em $25 \%$ dos menores de 6 meses, com tendência ascendente até os 2 anos. As maiores prevalências foram registradas no segundo ano de vida $(50,4 \%)$. Após os 24 meses esse percentual caiu para $20,3 \%$. O teste do $\mathrm{X}^{2}$ mostrou assossiação significante $(P<0,001)$ entre a presença de anemia e a faixa etária da criança, e a partição do $\mathrm{X}^{2}$ mostrou que a prevalência é maior entre 9 e 23 meses de idade.

Durante a realização do trabalho de campo, grande número de mães não retornou, conforme solicitado, após os 30 dias de suplementação. Dos 620 lactentes e pré-escolares que iniciaram o trabalho, apenas 289 compareceram esponta-

Tabela 1. Distribuição da população estudada por unidade de saúde e faixa etária.

\begin{tabular}{|c|c|c|c|}
\hline \multirow{2}{*}{$\begin{array}{l}\text { Faixa } \\
\text { Etária } \\
\text { (meses) }\end{array}$} & \multicolumn{3}{|c|}{ Unidade Básica de Saúde } \\
\hline & $\begin{array}{c}\text { Sta. Cecília } \\
(\mathrm{N})\end{array}$ & $\begin{array}{l}\text { Pari } \\
(\mathrm{N})\end{array}$ & $\begin{array}{c}\text { Total } \\
(\mathrm{N})\end{array}$ \\
\hline $\begin{array}{r}4 \text { e } 5 \\
6 \text { a } 8 \\
9 \text { a } 11 \\
12 \text { a } 17 \\
18 \text { a } 23 \\
24 \text { a } 36\end{array}$ & $\begin{array}{l}46 \\
42 \\
45 \\
63 \\
66 \\
66\end{array}$ & $\begin{array}{l}30 \\
55 \\
56 \\
60 \\
39 \\
52\end{array}$ & $\begin{array}{r}76 \\
97 \\
101 \\
123 \\
105 \\
118\end{array}$ \\
\hline Total & 328 & 292 & 620 \\
\hline
\end{tabular}


Tabela 2. Crianças com níveis de hemoglobina inferiores e superiores a $11,0 \mathrm{~g} / \mathrm{dl}$, segundo as faixas etárias estudadas. Número amostral $(\mathrm{N})$ percentagem e testes estatísticos.

\begin{tabular}{rcccc}
$\begin{array}{c}\text { Faixa } \\
\text { Etária }\end{array}$ & Amostra & $\begin{array}{c}\mathrm{Hb} \geq 11,0 \\
(\mathrm{~g} / \mathrm{dl})\end{array}$ & \multicolumn{2}{c}{$\begin{array}{c}\mathrm{Hb}<11,0 \\
(\mathrm{~g} / \mathrm{dl})\end{array}$} \\
\cline { 2 - 5 } (meses) & $(\mathrm{N})$ & $(\mathrm{N})$ & $(\mathrm{N})$ & $(\%)$ \\
\hline$<6(1)$ & 76 & 57 & 19 & 25,0 \\
$6 \geq 8(2)$ & 97 & 67 & 30 & 30,9 \\
$9 \geq 11(3)$ & 101 & 51 & 50 & 49,5 \\
$12 \geq 23(4)$ & 228 & 113 & 115 & 50,4 \\
$24 \geq 36(5)$ & 118 & 94 & 24 & 20,3 \\
\hline Total & 620 & 382 & 238 & 38,4 \\
\hline
\end{tabular}

$\mathrm{X}^{2}$ calculado $=43,57(\mathrm{P}<0,001)$

Participação do $X^{2}(3,4)>(1,2,5)$

neamente para a segunda avaliação (Tabela 3 ). Retornaram $200(52,4 \%)$ das crianças que não apresentavam anemia contra $89(37,4 \%)$ das anêmicas. O teste do $\mathrm{X}^{2}$ mostrou que é significantemente maior o percentual de retorno espontâneo em crianças não anêmicas $(P<0,001)$.

Como existiam componentes da amostra com hemoglobinas muito baixas, decidiu-se convocar, de casa em casa, todos os beneficiários do projeto que apresentam níveis inferiores a $11,0 \mathrm{~g} /$ dl. Dos 149 que foram convocados, apenas 68 atenderam ao chamado. Uma segunda coleta de sangue para dosagem da hemoglobina foi realizada em 155 crianças que apresentavam anemia e em 144 não anêmicos.

A Tabela 4 mostra que $56,3 \%$ das mães de crianças sem anemia administraram corretamente o fármaco a seus filhos, contra $49,0 \%$ das anêmicas. $\mathrm{O}$ teste do $\mathrm{X}^{2}$ não mostrou assossiação significante entre a administração do medicamento e a presença ou não de anemia.

A Tabela 5 registra a evolução dos níveis de hemoglobina nos momentos inicial e final da intervenção. Os testes de Kappa $(\mathrm{P}<0,01)$ e McNemar $(P<0,01)$ mostraram diminuição significante do número de crianças que apresentavam níveis de hemoglobina inferiores a $11,0 \mathrm{~g} /$ dl. Por outro lado, o total de crianças com hemoglobinas acima de $12,0 \mathrm{~g} / \mathrm{dl}$ subiu de 40 , no

Tabela 3. Distribuição das crianças estudadas, segundo suas condiçōes hematológicas, em funçāo de seus retornos às unidades básicas de saúde. Número amostral $(\mathrm{N})$, percentagem e testes estatísticos.

\begin{tabular}{ccccc}
\hline Aderência & \multicolumn{2}{c}{$\mathrm{Hb}<11,0 \mathrm{~g} / \mathrm{dl}$} & \multicolumn{2}{c}{$\mathrm{Hb} \geq 11,0 \mathrm{~g} / \mathrm{dl}$} \\
\cline { 2 - 5 } & $\mathrm{N}$ & $\%$ & $\mathrm{~N}$ & $\%$ \\
\hline $\begin{array}{c}\text { Retorno } \\
\begin{array}{c}\text { Espontâneo } \\
\text { Não } \\
\text { Retornou }\end{array}\end{array}$ & 89 & 37,4 & 200 & 52,4 \\
\hline Total & 238 & 62,6 & 182 & 47,6 \\
\hline $\mathrm{X}^{2}$ calculado $=$ & $13,19(\mathrm{P}<0,001)$ & & \\
\hline
\end{tabular}

Tabela 4. Distribuição das crianças estudadas, segundo suas condições hematológicas, em função da administração do medicamento. Número amostral (N), percentagem e teste estatistico.

\begin{tabular}{ccccc}
\hline Administração & \multicolumn{2}{c}{$\mathrm{Hb}<11,0 \mathrm{~g} / \mathrm{dl}$} & \multicolumn{2}{c}{$\mathrm{Hb} \geq 11,0 \mathrm{~g} / \mathrm{dl}$} \\
\cline { 2 - 5 } do medicamento & $\mathrm{N}$ & $\%$ & $\mathrm{~N}$ & $\%$ \\
\hline Correta & 76 & 49,0 & 81 & 56,3 \\
Incorreta & 65 & 41,9 & 51 & 35,4 \\
Duvidosa & 14 & 9,1 & 12 & 8,3 \\
\hline Total & 155 & 100,0 & 144 & 100,0 \\
\hline
\end{tabular}

$\mathrm{X}^{2}$ calculado $=1,60(\mathrm{NS})$

início, para 100, ao final do trabalho.

As médias das hemoglobinas nos dois momentos do estudo mostram comportamentos distintos em relação às faixas etárias estudadas, conforme indicado na Tabela 6. Em menores de 8 meses, não foram detectadas alterações significantes pelo teste do $\mathrm{t}$ pareado. Acima desta idade, registraram-se aumentos significantes das médias das hemoglobinas $(\mathrm{P}<0,001)$. O maior incremento foi observado entre os 24 e os 36 meses $(0,95 \mathrm{~g} / \mathrm{dl})$, seguido dos 18 aos 23 meses $(0,91 \mathrm{~g} / \mathrm{dl})$.

Na Figura relacionou-se a presença de anemia nos momentos anterior e posterior à intervação, com a administração do medicamento. Verificou-se que quando a mãe administrou corretamente o fármaco, a anemia caiu de $48,4 \%$ no início, para $28,1 \%$ ao final da intervenção. Se a administração se dava de forma incorreta essa queda era de $58,6 \%$ para $43,1 \%$. O teste de McNemar mostrou que essas reduções foram significantes tanto no grupo que recebeu corretamente o medicamento como naqueles que receberam de forma incorreta. $O$ teste do $\mathrm{X}^{2}$ não mostrou diferenças entre as reduções encontradas nos dois grupos analisados.

Em $80,3 \%$ das crianças não se regitrou qualquer efeito colateral ao medicamento. Em 37

Tabela 5. Distribuição das crianças estudadas, segundo os níveis de hemoglobina, nos momentos inicial e final da intervenção e testes estatísticos.

\begin{tabular}{|c|c|c|c|c|c|}
\hline \multirow{2}{*}{$\begin{array}{l}\text { Momento } \\
\text { Inicial } \\
\mathrm{Hb}(\mathrm{g} / \mathrm{dl})\end{array}$} & \multicolumn{5}{|c|}{ Momento final } \\
\hline & $<9,5$ & $9,5 \geq 10,9$ & $11,0 \geq 11,9$ & $\geq 12,0$ & Total \\
\hline $\begin{array}{c}<9,5 \\
9,5 \geq 10,9 \\
11,0 \geq 11,9 \\
\geq 12,0\end{array}$ & $\begin{array}{c}18 \\
6 \\
0 \\
0\end{array}$ & $\begin{array}{c}25 \\
40 \\
13 \\
3\end{array}$ & $\begin{array}{c}7 \\
43 \\
35 \\
9\end{array}$ & $\begin{array}{c}1 \\
15 \\
56 \\
28\end{array}$ & $\begin{array}{c}51 \\
104 \\
104 \\
40\end{array}$ \\
\hline Total & 24 & 81 & 94 & 100 & 299 \\
\hline \multicolumn{6}{|c|}{$\begin{array}{l}\text { Teste de Kappa }-\mathrm{K} w=0,38-\text { Zcalc. }=5,89 \cdot(\mathrm{P}<0,01) \\
\% \text { de melhorados }=40,47 \% \\
\% \text { de piorados }=10,37 \% \\
\% \text { de inalterados }=49,16 \% \\
\text { Teste de McNemar }-\mathrm{X}^{2} \text { calculado }=75,60(\mathrm{P}<0,001)\end{array}$} \\
\hline
\end{tabular}


Tabela 6. Valores de hemoglobinas das crianças estudadas, segundo faixas etárias, nos momentos inicial e final da intervenção. Médias ( $x$ ), desvios-padrão (s) e testes estatísticos.

\begin{tabular}{|c|c|c|c|c|c|c|}
\hline \multirow{2}{*}{$\begin{array}{c}\text { Faixa } \\
\text { etária } \\
\text { (meses) }\end{array}$} & \multirow{2}{*}{$\begin{array}{c}\text { Amostra } \\
\text { (N) }\end{array}$} & \multicolumn{4}{|c|}{$\begin{array}{c}\text { Média das Hemoglobinas } \\
(\mathrm{g} / \mathrm{dl})\end{array}$} & \multirow[t]{2}{*}{$\begin{array}{l}\text { Teste } t \\
\text { pareado }\end{array}$} \\
\hline & & $\begin{array}{c}\text { inicial } \\
(\mathrm{x})\end{array}$ & $\begin{array}{c}\text { inicial } \\
(\mathrm{s})\end{array}$ & $\begin{array}{c}\text { final } \\
(x)\end{array}$ & $\begin{array}{c}\text { final } \\
(\mathrm{s})\end{array}$ & \\
\hline$<6$ & 31 & 11,05 & 0,78 & 11,00 & 0,93 & NS \\
\hline $6 \geq 8$ & 35 & 10,93 & 1,01 & 11,11 & 1,04 & NS \\
\hline $9 \geq 11$ & 59 & 10,23 & 1,48 & 11,03 & 1,54 & $(P<0,001)$ \\
\hline $12 \geq 17$ & 71 & 10,32 & 1,40 & 11,10 & 1,23 & $(P<0,001)$ \\
\hline $18 \geq 23$ & 54 & 10,35 & 1,28 & 11,20 & 1,26 & $(P<0,001)$ \\
\hline $24 \geq 36$ & 49 & 11,12 & 1,15 & 12,10 & 1,20 & $(P<0,001)$ \\
\hline Total & 299 & 10,60 & 1,30 & 11,28 & 1,29 & \\
\hline
\end{tabular}

$(12,4 \%)$, as mães referiram diarréia e em 10 $(3,3 \%)$, fezes escuras.

\section{Comentários e Conclusões}

O processo de instalação da anemia nutricional por carência de ferro na população brasileira de menores de 2 anos inicia-se muito antes do nascimento. No Estado de São Paulo, a prevalência de anemia em gestantes varia entre 18,2 e $32,7 \%$, segundo os dados registrados nos arquivos da Secretaria de Estado da Saúde. Trabalhos recentes têm demostrado que recém-nascidos de mães anêmicas apresentam valores médios de hemoglobina significantemente inferiores aos apresentados pelos nascidos de mães não anêmicas ${ }^{21}$.

Outros fatores assossiados à etiopatogenia da anemia nutricional, em fases posteriores da vida, são a prematuridade e o baixo peso ao nascer, em função das baixas reservas de ferro acumuladas durante a gestação. Tanto a prematuridade como baixo peso ao nascer são comumente observados em recém-nascidos de

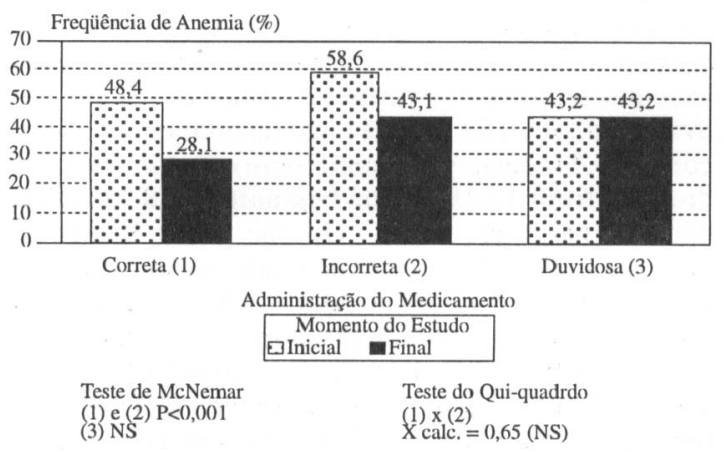

Figura. Prevalecências de anemia, nos momentos inicial e final do estudo, segundo a administração do medicamento e testes estatísticos. mães adolecentes e nas UBS do Estado de São Paulo elas representam 18\% do total das gestantes atendidas no serviço de pré-natal. Além disso, a multiparidade com intervalo interpartal curto impossibilita a reposição das perdas nutricionais da gestação anterior e, deste modo, pode desencadear a anemia na gestante e, conseqüentemente, no recém-nascido.

Após o nascimento é o leite materno que propicia ao lactente uma oferta adequada de ferro com elevada biodisponibilidade, sendo, portanto, o aleitamento natural fundamental para a manutenção de adequados níveis de hemoglobina nos primeiros meses de vida. Entretanto, o tempo de aleitamento materno médio, no Município de São Paulo, que em 1988 era 3,6 meses $^{12}$, hoje não chega a 2 meses, segundo levantamentos da Secretaria de Estado da Saúde de São Paulo. O leite de vaca, na forma líquida ou em pó, que além de apresentar baixa biodisponobilidade do mineral e provocar microhemorragias no trato gastrointestinal, acaba tornando-se a base da alimentação infantil nos dois primeiros anos de vida, elevando a ocorrência da anemia.

Todas essas colocações podem justificar os dados ora apresentados, pois, mesmo antes de completar 6 meses, $25 \%$ das crianças já apresentam níveis de hemoglobina inferiores a $11,0 \mathrm{~g} /$ dl, e a manutenção de uma dieta, essencialmente láctea, explica a prevalência de $50 \%$ de anemia encontrada entre os 9 e 23 meses. Finalmente, após os dois anos, quando a criança passa a receber uma dieta mais diversificada com maior oferta desse mineral é que se nota acentuada redução nos níveis de anemia.

O fraco vínculo mãe/filho tem sido referido como um dos fatores mais importantes na gênese da Desnutrição Energético-Protéica (DEP). Hoje, trabalhos desenvolvidos no Centro de Refêrencia de Saúde da Mulher, Nutrição, Alimentação e Desenvolvimento Infantil têm demostrado que esta situação decorre de uma série de alterações psicológicas maternas, reflexos de sua estória de vida e que acaba repercutindo na interação mãe/ filho, com importantes manifestações no campo nutricional ${ }^{14}$. Sem dúvida, estes aspectos justificam o fato da DEP ser encontrada ou não em populações com características socioeconômicas comuns como, renda familiar, tipo de moradia, meio ambiente e acesso às informações de saúde. Pelas respostas obtidas no presente trabalho, podemos concluir que a anemia carencial ferropriva não foge a esta regra e, desta forma, explicamos o grande número de mães que não retornaram, mesmo após visita domiciliar, para a segunda avaliação, bem como, o maior contigente 
de retorno de mães de crianças não anêmicas. Neste último caso, esta atitude é reflexo da preocupação da mãe com a saúde da criança, o que caracteriza um vínculo mãe/filho mais satisfatório.

A ignorância e o baixo nível de educação em saúde e nutrição são, também, reportados como causas da situação atual da anemia carencial em nossa população. Entretanto, as mães das crianças acompanhadas no presente estudo, foram esclarecidas, sobre a magnitude do problema e a importância da suplementação medicamentosa com sulfato ferroso. Conseqüentemente, apesar de igualadas do ponto de vista da informação, as respostas diferiram significantemente de mãe para mãe, o que vem reforçar os aspectos anteriormente assinalados. Quando perguntadas sobre o motivo que as levaram à administração do sulfato ferroso de forma incorreta, a resposta era o esquecimento. Concordamos que a obrigatoriedade de administrar diariamente um medicamento por longos períodos leva à saturação e ao esquecimento. Entretanto, as mães que não "esqueciam" de dar o sulfato ferroso, deveriam ser as mais preocupadas com a saúde e, portanto, com maior vínculo com seus filhos.

O decréscimo da ocorrência de anemia antes e após a suplementação medicamentosa com sulfato ferroso mostrou melhora significante, principalmente quanto à redução do número de crianças com $\mathrm{Hb}<11,0 \mathrm{~g} / \mathrm{dl}$, e o aumento daquelas com $\mathrm{Hb}>=12,0 \mathrm{~g} / \mathrm{dl}$. Estes fatos ocorreram apesar do sulfato ferroso ser prescrito em dose profilática (12 $\mathrm{mg} /$ dia), por um período de 30 dias e com apenas $50 \%$ das mães administrando-o corretamente. A diferença observada entre a redução da anemia nas crianças que receberam a suplementação conforme o prescrito (queda de 23 pontos percentuais) e de forma incorreta (queda de 15,5 pontos percentuais) confirma a eficácia deste tipo de intervenção.

A evolução das médias das hemoglobinas, antes e após a intervenção, analisada por faixa etária, também registra fatos dignos de consideração: as baixas respostas em menores de $6 \mathrm{e}$ entre 6 e 8 meses, com incrementos significantes a partir dos 9 meses. Explicam, essa diferença, as elevadas prevalências, onde pequenas doses de ferro podem representar um aporte rezoável para um organismo ávido pelo mineral. No conjunto, o comportamento das médias das hemoglobinas concorda com as conclusões obti-

* M. A. A. Torres e colaboradores - Estudo comparativo do efeito do uso do leite fortificado com ferro e vitamina C sobre os níveis de hemoglobina e condição nutricional de crianças menores de 2 anos em creches municipais e unidades básicas de saúde. Dados inéditos. das com os níveis de hemoglobina e a administração do medicamento.

Por fim, a suplementação medicamentosa através da administração diária de $12 \mathrm{mg} / \mathrm{dia}$ de ferro elementar, sob forma de sulfato ferroso, por um período de 30 dias, mostrou-se um tipo de intervenção eficaz do ponto de vista de melhoria dos níveis de hemoglobina nas crianças acompanhadas. Entretanto, do ponto de vista tanto operacional, como de saúde pública, a baixa anuência das mães à resposta leva a concluir que esta não seja a melhor forma de combater a anemia carencial ferropriva na população infantil brasileira. Dentro desta visão, a fortificação de alimentos tem se mostrado como eficiente caminho para a rápida resolução do problema*.

\section{Agradecimentos}

Ao Dr. Marcos Antonio Gonçalves Munhoz e à biologista Elena Yoko Gushiken pela realização dos exames laboratoriais; ao Cícero Alonso Canheta, pela coleta do sangue.

TORRES, M. A. A. et al. [Treatment with prophylactic doses of ferrous sulphate in the fight against iron deficiency in children attended in basis health units]. Rev. Saúde Pública, 28: 410-5, 1994. The efficacy of medical prophylaxis in the fight against iron deficiency anemia in 620 children aged from four to thirty-six months, attended in two health units of the municipality of S. Paulo, Brazil, was tested. First, the children's blood was tested for hemoglobin level and eletrophoresis. Then they received daily doses of iron sulphate in the form of drops, corresponding to $12 \mathrm{mg}$ / day of elementary iron, over a 30-day period. Afterwaeds, they were asked to return for re-evaluation. Twenty-five percent of children under 6 months presented hemoglobin levels under $11.0 \mathrm{~g} / \mathrm{dl}$. The highest occurrences of anemia were detected among children aged 9 to 11 months $(49.5 \%)$ and in the age bracket between 12 and 23 months (50.4\%). After the established period, only $37.4 \%$ of the children with anemia and $52.4 \%$ of children who did not present anaemia returned for further evaluation. Of the 299 evaluated, ony $157(52.5 \%)$ had taken the medication correctly. Frequency of hemoglobin inferior to $9.5 \mathrm{~g} / \mathrm{dl}$ dropped from $17.1 \%$ at the beginning to $8.1 \%$ at the end of the intervention. On the other hand, the percentage of children with hemoglobin above $12.0 \mathrm{~g} /$ dl increased from $13.4 \%$ to $33.4 \%$. Those chidren who took their iron supplements correctly registered a siginificantly higher drop in the number of cases of anemia than that observed in those who had not taken the supplement correctly. The great majority $(80.3 \%)$ did not present any side effects of medication. It was concluded that medical prophylaxis, instead of proving to be efficient in the recovery of hemoglobin levels, 
presents serious hazards from the operation point of view including a low level of participation in the project on the part of mothers, resulting from the daily obligation to administer a medication to their chidren over a long period of time which can consequence generate saturation and forgetfulness.

Keywords: Anemia, hypochromic, prevention \& control. Ferrous sulphate.

\section{Referências Bibliográficas}

1. ARAÚJO, J. T.; RIBEIRO, V. S.; BODEMEIER, L. Aspectos de laboratório da anemia drepanocítica (Anemia Falciforme). Laes Haes, 57: 16-23, 1989.

2. BATISTA-FILHO, M. \& BARBOSA, N. P. Alimentação $e$ nutriçāo infantil no Brasil: 1974-1984; PróMemória. Brasília, Ed. e Gráfica Canadá, 1985.

3. BRANDALISE, S. R. \& MATSUDA, E. Anemias carenciais. In: Nóbrega, F. J. Desnutrição intra-uterina e pós-natal. São Paulo, Panamed Ed., 1986. p. 427-37.

4. DALANG, G. A. \& CASTLE, W. B. A simples and rapid method for demonstrating sickling of the red blood cels: the use of reducing agents. J. Lab. Clin. Med., 3: $1082,1948$.

5. DeMAYER, E. M. Preventing and controlling iron deficiency anaemia through primary care. Ginebra, World Health Organization, 1989.

6. HAILINE, A. Standard methods of clinical chemistry. New York, Academic Press, v.2, p.52. 1958.

7. HERBART, V. Recomended dietary intakes (RDI) of iron in humans. Am. J. Clin. Nutr., 45: 679-86, 1987.

8. INTERNATIONAL COMMITEE FOR STANDARTIZATION IN HEMATOLOGY. Standard techniques for measurement of red cell and plasma volume. Br.J. Haematol., 25: 801, 1973.

9. JOÃO, W. S. J. Prevalência de anemia na população atendida nos postos de saúde da Secretaria de Saúde do Estado do Pará e em escolares participantes do Programa Nacional de Alimentação Escolar; relatório anual. Belém, Convênio INAN/UFPA, 1983.

10.LANDIS, J. R. \& KOCK, G. G. The measurement of observe agreement for categorical data. Biometrics, 33: $159.74,1977$.
11.LAUDERBACK, A. L.; YOUHNE, Y.; FONTANA, A. A.; NATHAND, M. Clinical evaluation of a rapid screening test of sickle cell trait (S-) and sickle cell anemia (SS). Clin. Chem. 20: 761-4, 1974.

12. MONTEIRO, C. A. Saude e nutrição das crianças de São Paulo: diagnóstico, contrastes sociatis e tendências. São Paulo, Hucitec/Ed. USP, 1988.

13. NESTLÉ NUTRITION. El hierro en la alimentación del lactante y del niño. s.l., 1984.

14. NÓBREGA, F. J. \& CAMPOS, A. L. R. Fraco vínculo mãe/filho: importante fator de risco de desnutrição. Ars Curandi, 26: 47-57, 1993.

15. ORGANIZACIÓN MUNDIAL DE LA SALUD. Lucha contra la anemia nutricional especialmente contra la carencia de hierro. Ginebra, 1975. (OMS - Série de Informes Técnicos, 580).

16.ORGANIZACIÓN MUNDIAL DE LA SALUD. Medición del efecto nutricional de programas de suplementación alimentaria a grupos vulnerables. Ginebra, 1980.

17. ROMANI, S.A. M.; LIRA, P. I.; BATISTA FILHO, M.; SEQUEIRA, L. A. S.; FREITAS, C. L. C. Anemias em pré-escolares: diagnóstico, tratamento e avaliação. RecifePe, Brasil. Arch. Latinoam. Nutr., 67: 159-67, 1991.

18. SIEGEL, S. Estadistica no parametrica. México, Ed. Trillas, 1975.

19.SIGULEN, D. M.; TUDISCO, E. S.; GOLDEMBERG, T.; ATHAIDE, M. M. M.; VAISMAN, E. Anemia ferropriva em crianças no Município de São Paulo. Rev. Saúde Pública, 12: 168-78, 1978.

20.SOKAL, R. R. \& ROHLF, F. J. Biometry. San Francisco, W. H. Freeman and Company, 1969.

21.SZARFARC, S. C. Comparação entre os valores hematológicos (hemoglobina, hematócrito e ferro sérico) da parturiente e do recém-nascido. Rev. Saúde Pública, 9: 43-7, 1975.

22. TORRES, M. A. A. Estado nutricional e aspectos socioeconômicos de famílias rurais do Trópico semi-árido (Nordeste do Brasil). Recife, 1982. [Dissertação de Mestrado - Universidade Federal de Pernambuco].

23.TORRES, M. A. A.; SATO, K.; SOUZA QUEIROZ, S. Anemia em crianças menores de 2 anos atendidas nas unidades básicas de saúde no Estado de São Paulo. Rev. Saúde Pública, 28(4) :290-4, 1994.

Recebido para publicação em 17.2.1994 Reapresentado em 22.6.1994 Aprovado para publicação em 23.9.1994 